\title{
CRESCIMENTO INICIAL DE PITAIA EM FUNÇÃO DE COMBINAÇÕES DE DOSES DE FÓSFORO-ZINCO ${ }^{1}$
}

\author{
MÁRCIO CLEBER DE MEDEIROS CORRÊA ${ }^{2,6}$, EDMILSON IGOR BERNARDO ALMEIDA ${ }^{3}$ \\ VIRNA BRAGA MARQUES ${ }^{4}$, JÚLIO CÉSAR DO VALE SILVA \\ BOANERGES FREIRE DE AQUINO ${ }^{6}$
}

\begin{abstract}
RESUMO - Com o objetivo de avaliar a interferência da relação fósforo-zinco sobre o crescimento inicial de pitaia para fornecer informações que subsidiem a definição de sistemas de produção mais adequados para a exploração desta cultura no Brasil, realizou-se o experimento, testando cinco doses de $\mathrm{P}(0 ; 75 ; 150$; 225 e $\left.300 \mathrm{mg} \mathrm{dm}^{-3}\right)$ e $\mathrm{Zn}\left(0 ; 2 ; 4 ; 6\right.$ e $\left.8 \mathrm{mg} \mathrm{dm}^{-3}\right)$, sendo os níveis de cada nutriente arranjados em esquema fatorial $5 \times 5$, com quatro blocos. Para a interação significativa $(p<0,05)$, procedeu-se ao ajuste em superfícies de resposta do tipo $\mathrm{Y}=\beta_{0}+\beta_{1}(\mathrm{~A})+\beta_{2}(\mathrm{~A})^{2}+\beta_{3}(\mathrm{~B})+\beta_{4}(\mathrm{~B})^{2}+\beta_{5}(\mathrm{~A})$. (B) $+e$. As estimativas das correlações fenotípicas foram obtidas considerando apenas o intervalo das doses de $\mathrm{Pe} Z \mathrm{Zn}$ que promoveram as melhores respostas do acesso de pitaia para os caracteres estudados. A aplicação de P e Zn, e a interação P x Zn afetam a disponibilidade de ambos os nutrientes no substrato, sistema radicular e parte aérea, influenciando, assim, o crescimento inicial de plantas de pitaia. Os rendimentos mais satisfatórios ocorrem quando há $60-75$ $\mathrm{mg}$ de $\mathrm{P} \mathrm{dm}{ }^{-3}$ e 3,0 - 4,0 mg de $\mathrm{Zn} \mathrm{dm}{ }^{-3}$ no substrato, e 4,5-6,0 $\mathrm{g} \mathrm{kg}^{-1}$ de $\mathrm{P}$ e $150 \mathrm{mg}$ de $\mathrm{Zn} \mathrm{kg}^{-1}$ na parte

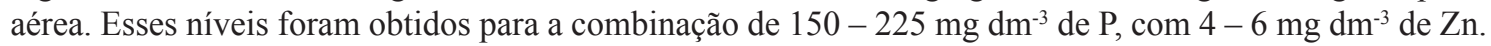
Os teores de $\mathrm{P}$ e Zn na parte aérea e o somatório do comprimento dos cladódios (SCC) são os caracteres explicativos que apresentam maiores efeitos diretos sobre a massa seca dos cladódios (MSC).

Termos para indexação: adubação, Cactaceae, frutífera exótica, Hylocereus undatus, interação entre nutrientes.
\end{abstract}

\section{EARLY GROWTH OF DRAGON FRUIT DUE TO COMBINATIONS OF PHOSPHORUS-ZINC}

\begin{abstract}
With the aim to evaluate the role of zinc-phosphorus ratio in the initial growth of pitaia for provide information that supports the definition of production systems more suitable for the exploitation of this crop in Brazil an experiment was carried out, testing five levels of $\mathrm{P}\left(0,75,150,225\right.$ and $\left.300 \mathrm{mg} \mathrm{dm}^{-3}\right)$ and $\mathrm{Zn}\left(0,2,4,6\right.$ and $\left.8 \mathrm{mg} \mathrm{dm}^{-3}\right)$ were each nutrient levels, arranged in $5 \times 5$ factorial design with four blocks. For significant interaction $(p<0.05)$ proceeded adjustment in response surfaces of type $Y=\beta_{0}+\beta_{1}(A)+\beta_{2}$ $(\mathrm{A})^{2}+\beta_{3}(\mathrm{~B})+\beta_{4}(\mathrm{~B})^{2}+\beta_{5}(\mathrm{~A})$. (B) + e. Estimates of phenotypic correlations were obtained considering only the range of doses of $\mathrm{P}$ and $\mathrm{Zn}$ that promoted the best answers to the access pitaya traits. Application of $\mathrm{P}$ and $\mathrm{Zn}$, and $\mathrm{P} \times \mathrm{Zn}$ interaction affect both the availability of nutrients in the soil, roots and shoots, thus influencing the early growth of pitaya. The most satisfactory yields occur when there is $60-75 \mathrm{mg} \mathrm{P} \mathrm{dm}^{-3}$ and $3.0-4.0 \mathrm{mg} \mathrm{dm}^{-3}$ of $\mathrm{Zn}$ in the soil and 4.5 to $6.0 \mathrm{~g} \mathrm{~kg}^{-1}$ and $150 \mathrm{mg}$ of P Zn kg${ }^{-1}$ in shoots. These levels were obtained for the combination of 150 to $225 \mathrm{mg} \mathrm{dm}^{-3}$ with P $4-6 \mathrm{mg} \mathrm{dm}^{-3} \mathrm{of} \mathrm{Zn}$. The levels of $\mathrm{P}$ and $\mathrm{Zn}$ in the shoot and the sum of the length of cladodes (SCC) are the characters that have greater explanatory direct effects on dry weight of cladodes (MSC).
\end{abstract}

Index terms: fertilization, Cactaceae, exotic fruit, Hylocereus undatus, interaction between nutrients.

'(Trabalho 297-13). Recebido em: 22-08-25013. Aceito para publicação em: 26-03-2014.

${ }^{2}$ Professor Adjunto do Departamento de Fitotecnia do Centro de Ciências Agrárias da Universidade Federal do Ceará (UFC), Campus do Pici: E-mail: mcleber@ufc.br

${ }^{3}$ Doutorando no Programa de Pós-Graduação em Agronomia: Fitotecnia, Campus do Pici, Universidade Federal do Ceará (UFC): E-mail: edmilson_i@hotmail.com

${ }^{4}$ Pós-Doutoranda no Programa de Pós-Graduação em Agronomia: Fitotecnia, Campus do Pici, Universidade Federal do Ceará (UFC): Evirnabm@hotmail.com

${ }^{5}$ Professor Adjunto do Departamento de Fitotecnia do Centro de Ciências Agrárias da Universidade Federal do Ceará (UFC), Campus do Pici: juliodovale@ufc.br

${ }^{6}$ Professor Associado do Departamento de Solos do Centro de Ciências Agrárias da Universidade Federal do Ceará (UFC), Campus do Pici: aquino@ufc.br 


\section{INTRODUÇÃO}

A pitaia (Hylocereus undatus) é uma cactácea epífita, perene, suculenta, que apresenta caule do tipo cladódio, de onde partem numerosas raízes adventícias que permitem o crescimento da planta em árvores e pedras situadas em ambientes sombreados de florestas tropicais da América. A pitaia está distribuída na Costa Rica, Venezuela, Panamá, Uruguai, Brasil, Colômbia e México, sendo os dois últimos países, os principais produtores, em nível mundial (ORTIZ-HERNÁNDEZ; CARRILLOSALAZAR, 2012; DONADIO, 2009).

De acordo com Lima et al. (2007), a interação fósforo-zinco é uma situação clássica que exprime a interferência de um elemento sobre o outro. Essa interação é bastante estudada, porém é um fenômeno complexo e pouco entendido, visto que existem diferentes resultados que sugerem a existência de antagonismo mútuo entre $\mathrm{P}$ e $\mathrm{Zn}$, quando ambos os nutrientes excedem seus valores críticos.

Com o objetivo de avaliar a interferência da relação fósforo-zinco sobre o crescimento inicial de pitaia, e com isso fornecer informações que subsidiem a definição de sistemas de produção mais adequados para a exploração desta cultura no Brasil, realizou-se o experimento.

\section{MATERIAL E MÉTODOS}

O experimento foi conduzido em casa de vegetação localizada no Setor de Horticultura do Departamento de Fitotecnia, Centro de Ciências Agrárias da Universidade Federal do Ceará (UFC), situado na cidade de Fortaleza - CE. O plantio das partes propagativas de pitaia foi realizado em vasos com capacidade para $12 \mathrm{dm}^{3}$, preenchidos com $10 \mathrm{dm}^{3}$ de substrato. O substrato foi amostrado e encaminhado ao laboratório, onde foi analisado quanto às suas características físicas e químicas. Ele apresentou as seguintes características: classificação textural - areia franca; composição química $\mathrm{pH}$ (água): 7,2; $\mathrm{Ca}, \mathrm{Mg}, \mathrm{K}, \mathrm{Na}, \mathrm{Al}^{3+}, \mathrm{H}+\mathrm{Al}$, respectivamente, 0,$40 ; 0,30 ; 0,20 ; 0,10 ; 0,0$ e 0,0 $\mathrm{mmol}_{\mathrm{c}} \mathrm{dm}^{-3} ; \mathrm{P}, \mathrm{Cu}, \mathrm{Fe}, \mathrm{Zn}$ e $\mathrm{Mn}$, respectivamente, 18,$0 ; 0,50 ; 30,16 ; 2,48$ e $2,26 \mathrm{mg} \mathrm{dm}^{-3}$; e matéria orgânica de $1,80 \mathrm{~g} \mathrm{~kg}^{-1}$.

Como materiais propagativos, utilizaram-se cladódios com três classes de comprimento: pequeno $(8-20 \mathrm{~cm})$, médio $(21-33 \mathrm{~cm})$ e grande $(34-46 \mathrm{~cm})$, havendo a distribuição de, pelo menos, dois cladódios de tamanho médio dentre as quatro repetições utilizadas para cada tratamento. A irrigação foi realizada manualmente. Para a manutenção do teor de umidade do substrato, em torno de $60 \%$, usaram-se béqueres contendo as quantidades de água calculadas. Por sua vez, o tutoramento dos vasos foi realizado aos 95 dias, tendo em vista o acentuado crescimento das plantas, a partir desse período.

Em virtude da ausência de recomendação de adubação para a cultura da pitaia, as doses foram estimadas com base em recomendação geral para experimento em vasos, boletins técnicos da cultura do abacaxi e experimentos conduzidos com palma forrageira. Foram testadas cinco doses de $\mathrm{P}(0,75$, 150,225 e $\left.300 \mathrm{mg} \mathrm{dm}^{-3}\right)$ e $\mathrm{Zn}\left(0,2,4,6\right.$ e $\left.8 \mathrm{mg} \mathrm{dm}^{-3}\right)$, sendo que os níveis de ambos os nutrientes foram arranjados em esquema fatorial $5 \times 5$, com quatro repetições. Os adubos utilizados como fonte de $\mathrm{P}$ e $\mathrm{Zn}$ foram, respectivamente, superfosfato triplo (45\% de P) e sulfato de zinco (20\% de Zn). Para a adubação básica (igual para todos os vasos), foram utilizados: $62,50 \mathrm{mg} \mathrm{dm}^{-3}$ de cloreto de potássio $375,00 \mathrm{mg} \mathrm{dm}^{-3}$ de sulfato de amônio, e 25,40 $\mathrm{mg} \mathrm{dm}^{-3}$ de FTE BR-11. À exceção do superfosfato triplo e do FTE BR-11, que foram aplicados em quantidade total, durante o preenchimento dos vasos, os demais adubos foram adicionados em cinco vezes iguais, solubilizados em água para favorecer sua incorporação ao substrato.

Aos 180 dias do plantio dos cladódios (cerca de 150 dias após a aplicação dos tratamentos), fizeram-se a análise fenológica das plantas, a coleta dos cladódios laterais e a amostragem do substrato utilizado para o preenchimento dos vasos. Os caracteres fenológicos avaliados foram os seguintes: somatório do comprimento dos cladódios (SCC), número de cladódios (NC), massa fresca dos cladódios (MFC), massa seca dos cladódios (MSC), comprimento do sistema radicular (CSR) e massa seca do sistema radicular (MSSR). Ao término das determinações da massa seca dos cladódios e do sistema radicular, o material foi moído para a determinação dos teores de $\mathrm{P}$ e $\mathrm{Zn}$ em ambos os compartimentos da planta. O substrato foi coletado em cada vaso, embalado em saco plástico, identificado e encaminhado para o laboratório, onde foi submetido à secagem em estufa com circulação forçada de ar, à temperatura de $50^{\circ} \mathrm{C}$, durante 24 horas. Em seguida, o material foi peneirado e analisado quanto ao teor de $\mathrm{P}$ e $\mathrm{Zn}$. Os resultados das variáveis químicas e fenológicas foram submetidos à análise de variância (teste $\mathrm{F}$ com nível de significância $\mathrm{p}<0,05$ ), de modo a avaliar os efeitos principais de cada uma das variáveis (A e B) e da interação (A x B). Para a interação significativa $(\mathrm{p}<0,05)$, procedeu-se ao ajuste em superfícies de resposta do tipo $\mathrm{Y}=\beta_{0}+$ $\beta_{1}(\mathrm{~A})+\beta_{2}(\mathrm{~A})^{2}+\beta_{3}(\mathrm{~B})+\beta_{4}(\mathrm{~B})^{2}+\beta_{5}(\mathrm{~A})$. ( B $)+e$, em que $\mathrm{Y}$ foi a variável de resposta; $\mathrm{A}$, dose de $\mathrm{A}(\mathrm{mg}$ 
$\left.\mathrm{dm}^{-3} \mathrm{de} A\right) ; \mathrm{B}$, dose de B (mg dm ${ }^{-3}$ de B $) ; \beta_{0}, \beta_{1}, \beta_{2}, \beta_{3}$, $\beta_{4}$ e $\beta_{5}$, os parâmetros do modelo, e $e$, o erro aleatório associado a cada observação.

As estimativas das correlações fenotípicas foram obtidas considerando apenas o intervalo das doses de $\mathrm{P}$ e $\mathrm{Zn}$ que promoveram as melhores respostas nas plantas de pitaia para os caracteres estudados. Posteriormente, foi realizada a diagnose de multicolinearidade entre os caracteres explicativos, e seu grau na matriz de correlação foi estabelecido com base no número de condição (razão entre o maior e o menor autovalor da matriz). O desdobramento das correlações fenotípicas, em efeitos diretos e indiretos dos caracteres explicativos sobre a massa seca de cladódios (caráter dependente), foi feito por meio da análise de trilha (Wright, 1921). As análises estatísticas e de trilhas foram executadas por meio do aplicativo computacional em Genética e Estatística, GENES.

\section{RESULTADOS E DISCUSSÃO}

A interação fósforo-zinco foi significativa $(\mathrm{p}<0,01)$ para o teor de $\mathrm{P}$ e $\mathrm{Zn}$ no substrato e na parte aérea, assim como para as variáveis fenológicas analisadas. Em razão disso, as médias das respectivas variáveis foram analisadas através de superfícies de resposta com modelo matemático do tipo $\mathrm{Y}=\beta_{0}+$ $\beta_{1}(\mathrm{P})+\beta_{2}(\mathrm{P})^{2}+\beta_{3}(\mathrm{Zn})+\beta_{4}(\mathrm{Zn})^{2}+\beta_{5}(\mathrm{P}) \cdot(\mathrm{Zn})+e$.

$O$ teor de $P$ no substrato variou de 5,40 a 75,38 $\mathrm{mg}$ de $\mathrm{P} \mathrm{dm}^{-3}$. Os menores valores $(<45,00 \mathrm{mg}$ de $\mathrm{P}$ $\mathrm{dm}^{-3}$ ) resultaram da aplicação de $0-75 \mathrm{mg} \mathrm{dm}^{-3} \mathrm{de}$ $\mathrm{P}$ com $4-8 \mathrm{mg} \mathrm{dm}^{-3} \mathrm{de} \mathrm{Zn}$, ao passo que as maiores

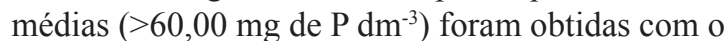
uso combinado de $225-300 \mathrm{mg} \mathrm{dm}^{-3} \mathrm{de} P$ com $2-4$ $\mathrm{mg} \mathrm{dm}{ }^{-3}$ de $\mathrm{Zn}$ (Figura 1A). Portanto, a combinação das maiores doses de $\mathrm{P}$ com as menores doses de $\mathrm{Zn}$ resultou no aumento da disponibilidade de $\mathrm{P}$ no substrato ao término do período experimental, o que evidencia um expressivo efeito antagônico do $\mathrm{Zn}$ sobre o P, quando esse micronutriente é utilizado em doses elevadas. Os maiores incrementos nas variáveis fenológicas ocorreram para $60-75 \mathrm{mg}$ de $\mathrm{P} \mathrm{dm}^{-3}$ no substrato. A aplicação de doses crescentes de $P$ provocou aumento linear no teor de $P$ disponível no substrato, independentemente da adubação com zinco (Figura 1A).

$\mathrm{O}$ teor de $\mathrm{P}$ na parte aérea variou de 1,64 a 7,73. Os menores valores $\left(<4,50 \mathrm{~g} \mathrm{~kg}^{-1}\right)$ foram resultantes da aplicação de $0-75 \mathrm{mg} \mathrm{dm}^{-3}$ de $\mathrm{P}$ com $2-8 \mathrm{mg} \mathrm{dm}^{-3}$ de $\mathrm{Zn}$. As maiores médias $(>6,00 \mathrm{~g}$ $\mathrm{kg}^{-1}$ ) foram obtidas com o uso de $150-300 \mathrm{mg} \mathrm{dm}^{-3}$ de P com 2-8 $\mathrm{mg} \mathrm{dm}^{-3}$ de Zn (Figura 1B). Houve incremento no teor $\mathrm{P}$ da parte aérea de pitaia com o aumento da disponibilidade desse elemento no substrato (Figura 1B). Essas observações estão de acordo com os resultados apresentados por Batista et al. (2011), Cessa et al. (2009) e Fernandes et al. (2007) para mudas de goiabeira, capuchinha e freijó, respectivamente. $\mathrm{O}$ aumento da disponibilidade de $\mathrm{P}$ no substrato ocasionou efeito positivo sobre o crescimento vegetativo, o qual possivelmente induziu o aumento do teor de P na parte aérea. Os maiores incrementos vegetativos foram obtidos para um teor de 4,5-6,0 $\mathrm{g} \mathrm{kg}^{-1}$ de $\mathrm{P}$ na parte aérea.

$\mathrm{O}$ teor de $\mathrm{Zn}$ no substrato variou de 0,57 a $9,38 \mathrm{mg} \mathrm{dm}^{-3}$, sendo que os menores valores $(<4,00$ $\mathrm{mg} \mathrm{dm}{ }^{-3}$ ) foram obtidos com a aplicação de $225-300$ $\mathrm{mg} \mathrm{dm}{ }^{-3}$ de $\mathrm{Pe} 0-4 \mathrm{mg} \mathrm{dm}^{-3} \mathrm{de} \mathrm{Zn}$, ao passo que os maiores teores $\left(>5,00 \mathrm{mg} \mathrm{de} \mathrm{Zn} \mathrm{dm}^{-3}\right)$ resultaram do uso de $0-150 \mathrm{mg} \mathrm{dm}^{-3}$ de P com 6-8 $\mathrm{mg} \mathrm{dm}^{-3} \mathrm{de} \mathrm{Zn}$ (Figura 1C). Portanto, a utilização de doses elevadas de P (225-300 $\left.\mathrm{mg} \mathrm{dm}^{-3}\right)$ com doses baixas de $\mathrm{Zn}(0$ $4 \mathrm{mg} \mathrm{dm}^{-3}$ ) provocou redução no teor de Zn disponível no substrato, o que demonstra efeito antagônico do $\mathrm{P}$ quando em altas doses sobre o $\mathrm{Zn}$ e confirma os resultados apresentados por Muner et al. (2011) em milho. Segundo os autores, o uso de adubação fosfatada excessiva pode ocasionar deficiência de $\mathrm{Zn}$ no substrato, pois o fósforo insolubiliza o zinco na superfície das raízes ou precipita-o como fosfato de zinco, impedindo a absorção do nutriente pelas plantas. Os maiores incrementos vegetativos de pitaia foram verificados para um teor de 3,0-4,0 $\mathrm{mg}$ de $\mathrm{Zn} \mathrm{dm}^{-3}$ no substrato (Figura 1C).

$\mathrm{O}$ teor de $\mathrm{Zn}$ na parte aérea oscilou de 64,44 a $238,35 \mathrm{mg} \mathrm{dm}^{-3} \mathrm{de} \mathrm{Zn}$. As menores médias $\left(<100,00 \mathrm{mg} \mathrm{dm}^{-3} \mathrm{de} \mathrm{Zn}\right)$ resultaram da combinação de $225-300 \mathrm{mg} \mathrm{dm}^{-3}$ de P com $0-2 \mathrm{mg} \mathrm{dm}^{-3}$; ou $8 \mathrm{mg} \mathrm{dm}-3$ de Zn com $225-300 \mathrm{mg} \mathrm{dm}^{-3}$ de P. Por sua vez, os maiores teores $\left(>100,00 \mathrm{mg} \mathrm{dm}^{-3} \mathrm{de} \mathrm{Zn}\right.$ ) foram obtidos com o uso de $0-150 \mathrm{mg} \mathrm{dm}^{-3} \mathrm{de} P$ com 4 - $8 \mathrm{mg} \mathrm{dm}^{-3}$ de Zn (Figura 1D). Observou-se que, em condições de boa disponibilidade de $\mathrm{Zn}$ no substrato (>3,0 - 4,0 mg de Zn $\mathrm{dm}^{-3}$ no substrato) (Figura 1D), houve elevado acúmulo do elemento, tanto no sistema radicular quanto na parte aérea. De acordo com Sartori (2008) e Martinez (2005), o zinco possui redistribuição limitada na planta, mas em plantas bem supridas com o nutriente, a mobilidade no floema pode ser aumentada. Os maiores incrementos vegetativos foram constatados para um teor de aproximadamente $150 \mathrm{mg} \mathrm{de} \mathrm{Zn} \mathrm{kg}^{-1}$ na parte aérea.

O SCC variou de 81,75 a $176 \mathrm{~cm}$. Os menores valores $(<112,00 \mathrm{~cm})$ foram encontrados tanto para a testemunha quanto para a adição de $75 \mathrm{mg} \mathrm{dm}^{-3} \mathrm{de}$ P com 2- $4 \mathrm{mg} \mathrm{dm}^{-3} \mathrm{de} \mathrm{Zn}$. Por sua vez, os maiores 
valores $(>120,00 \mathrm{~cm})$ resultaram do uso de $150-300$ $\mathrm{mg} \mathrm{dm}{ }^{-3}$ de P com 6- $8 \mathrm{mg} \mathrm{dm}^{-3}$ de Zn (Figura 2A). A deficiência de $\mathrm{P}$ e Zn ocasiona déficit de ATP e redução na síntese de auxina e RNA, comprometendo o metabolismo das plantas e, consequentemente, a diminuição no crescimento vegetativo das mesmas, como observado nas plantas de pitaia que receberam os menores suprimentos de $\mathrm{P}$ e $\mathrm{Zn}$.

$\mathrm{O}$ número de cladódios $(\mathrm{NC})$ variou de $5,00-9,00$ unidades. Os menores resultados $(<6,00$ cladódios) foram obtidos para a testemunha e para a aplicação de $75 \mathrm{mg} \mathrm{dm}^{-3}$ de P com $2-4 \mathrm{mg} \mathrm{dm}^{-3}$ de $Z n$. Por sua vez, as maiores médias ( $>7,00$ unidades) resultaram da aplicação de $150-225 \mathrm{mg} \mathrm{dm}^{-3}$ de $\mathrm{P}$ com 6-8 $\mathrm{mg} \mathrm{dm}^{-3} \mathrm{de} \mathrm{Zn}$ (Figura 2B). Esses maiores valores ( $>7,00$ cladódios) corroboraram o NC obtido por Moreira et al. (2011) em plantas de pitaia; e com o número de artículos encontrados por Ramos (2011) e Teles et al. (2002) em plantas de palma forrageira. Possivelmente, o aumento da disponibilidade de $\mathrm{Pe}$ Zn no substrato influenciou a síntese de ATP, RNA e AIA nas plantas. Em razão disso, pode ter havido a formação de brotações laterais, que resultaram no aumento do número de cladódios.

A aplicação de $150-300 \mathrm{mg} \mathrm{dm}^{-3}$ de $\mathrm{P}$ com $4-8 \mathrm{mg} \mathrm{dm}^{-3}$ de $\mathrm{Zn}$ ocasionou aumento na disponibilidade de $\mathrm{P}$ e $\mathrm{Zn}$ no substrato, capaz de promover significativos incrementos no SCC e NC (Figuras 1A, 1C, 2A e 2B). De acordo com Muner (2011), plantas bem supridas em P e Zn apresentam expressivas médias de massa seca e produção. Contudo, plantas adubadas com $\mathrm{P}$, na ausência de aplicação de $\mathrm{Zn}$, demonstraram sinais de toxidez de $\mathrm{P}$, em virtude do acúmulo desse nutriente no tecido e da ineficiência de utilização pela planta, o que possivelmente está relacionado ao efeito sinegístico desempenhado pelo $\mathrm{Zn}$.

A massa seca dos cladódios oscilou de 20,00 a $36,25 \mathrm{~g}$. As menores médias $(<28,00 \mathrm{~g})$ resultaram da adição de $0-75 \mathrm{mg} \mathrm{dm}^{-3}$ de $\mathrm{P}$ com 0 - $8 \mathrm{mg} \mathrm{dm}^{-3}$ de $\mathrm{Zn}$, ao passo que os maiores valores $(>28,00 \mathrm{~g})$ foram obtidos com o uso de $150-300 \mathrm{mg} \mathrm{dm}^{-3} \mathrm{de}$ P com 2 - $6 \mathrm{mg} \mathrm{dm}^{-3}$ de Zn. Portanto, a adubação fosfatada exerceu papel limitante no incremento de MSC, sendo que os resultados mais expressivos $(>28,00 \mathrm{~g})$ foram proporcionados pelo uso de doses de $\mathrm{P}$ e $\mathrm{Zn}$ capazes de propiciar boa disponibilidade de ambos os nutrientes no substrato e na parte aérea das plantas. De acordo David et al. (2008), o Zn aumenta a eficiência de utilização de $P$ na parte aérea, podendo haver toxidez de doses elevadas de $\mathrm{P}$ em plantas deficientes em $\mathrm{Zn}$. A faixa que abrangeu as maiores médias de SCC, NC e MFC coincidiu com os maiores valores de MSC (Figuras 1A, 1B, 1C, 1D,

\section{A, 2B, 2C e 2D).}

Verificou-se que a MSC variou de 20,00 a 36,25 g, enquanto a MFC oscilou de 126,75 a 270,00 g (Figuras 2C e 2D). Relacionando-se ambos os resultados, estimou-se que, em torno de $84,00-87,00 \%$ do tecido vegetal estava constituído por água. Esses resultados corroboraram o teor de umidade descrito por Goldstein et al. (1991) para cactos suculentos.

O comprimento do sistema radicular oscilou de 35,25 a $53,00 \mathrm{~cm}$. Os menores valores $(<42,00$ $\mathrm{cm})$ resultaram da aplicação de $0 \mathrm{mg} \mathrm{dm}^{-3} \mathrm{de} P$ com $0-8 \mathrm{mg} \mathrm{dm}^{-3} \mathrm{de} \mathrm{Zn}$; ou $225-300 \mathrm{mg} \mathrm{dm}^{-3} \mathrm{de} P$ com $0-2 \mathrm{mg} \mathrm{dm}^{-3} \mathrm{de} \mathrm{Zn}$. Por sua vez, as maiores médias $(>42,00 \mathrm{~cm})$ foram obtidas com o uso de $75-225 \mathrm{mg}$ $\mathrm{dm}^{-3} \mathrm{de}$ P com 2 - $6 \mathrm{mg} \mathrm{dm}^{-3}$ de Zn (Figura 2E). Logo, constatou-se que tanto a zona de deficiência quanto a zona de elevada disponibilidade de P no substrato culminaram em baixos incrementos do comprimento do sistema radicular. De acordo com Corrêa (2004), o suprimento adequado de $\mathrm{P}$ ao substrato pode induzir o crescimento do sistema radicular, pois o $P$ é essencial para sua formação. Rosolem et al. (1999) citaram que, em condições de deficiência de P no solo, o elemento é preferencialmente concentrado nas raízes.

A massa seca do sistema radicular oscilou de 8,75 a $22,50 \mathrm{~g}$. Os menores valores resultaram da aplicação de $0-150 \mathrm{mg} \mathrm{dm}^{-3} \mathrm{de} \mathrm{P}$ e $2-8 \mathrm{mg} \mathrm{dm}^{-3}$ de $\mathrm{Zn}$, ao passo que as maiores médias foram obtidas com o uso de $225-300 \mathrm{mg} \mathrm{dm}^{-3}$ de P e 2 - $8 \mathrm{mg} \mathrm{dm}^{-3}$ de Zn. Esses resultados exprimiram o papel limitante que o P desempenha no crescimento do sistema radicular (Figura 2F). A aplicação da maior dose de $P$ estimulou o crescimento vegetativo em detrimento do sistema radicular, aumentando a relação parte área-raiz. Esses resultados corroboraram Almeida Júnior et al. (2009). Segundo esses autores, em condições de elevada disponibilidade de $\mathrm{P}$ no solo, as plantas têm possibilidade de investir maior parcela de energia no incremento da parte aérea. Possivelmente, a aplicação de doses de Zn na ausência da adubação fosfatada estimulou o crescimento lateral do sistema radicular, pois ao passo que incrementaram a MSSR, estas mesmas combinações ocasionaram redução linear no CSSR.

Com o intuito de conhecer os efeitos diretos e indiretos dos caracteres explicativos sobre a variável básica, massa seca dos cladódios, procedeu-se à análise de trilha. De acordo com Silva et al. (2005), a análise de trilha proporciona o detalhamento dos efeitos, complementando as informações dadas pela correlação. Para reduzir os efeitos de multicolinearidade e, desse modo, a atenuação de conclusões imprecisas, a análise de 
trilha foi construída, apenas com as combinações de doses de $\mathrm{P}$ e $\mathrm{Zn}$ que apresentaram os resultados mais satisfatórios, em termos de disponibilidade dos elementos no substrato e tecido vegetal, e de incremento das variáveis fenológicas avaliadas. De acordo com Coimbra (2005), entre os efeitos peculiares de uma elevada multicolinearidade, podem ser citadas a estimativa inconsistente do coeficiente de regressão e também uma superestimativa dos efeitos diretos das variáveis explicativas sobre a variável resposta, o que pode levar à interpretação equivocada.

Em síntese, as doses de $\mathrm{Pe} \mathrm{Zn}$, utilizadas para a execução da análise de trilha, foram as seguintes: 150 - $225 \mathrm{mg} \mathrm{dm}^{-3}$ de P com $4-6 \mathrm{mg} \mathrm{dm}^{-3}$ de $\mathrm{Zn}$, as quais resultaram em quatro combinações fósforozinco. As variáveis teor de $\mathrm{Pe} \mathrm{Zn}$ no substrato e MFC também foram excluídas da análise de trilha devido à mesma justificativa estatística (diminuir os efeitos de multicolinearidade) (Figuras 1 e 2). A interação fósforo-zinco foi significativa a $(p<0,05)$ pelo teste $\mathrm{F}$, para o teor de P na parte aérea, SCC, MSC, CSSR e MSSR. O teor de $\mathrm{Zn}$ na parte aérea foi influenciado, apenas pelas doses de $\mathrm{P}(\mathrm{p}<0,01)$, enquanto o $\mathrm{NC}$ sofreu efeito dos fatores $\mathrm{P}(\mathrm{p}<0,01)$ e $\mathrm{Zn}(\mathrm{p}<0,01)$, isoladamente.

O coeficiente de determinação $\left(\mathrm{R}^{2}\right)$ e o efeito residual indicam quanto das variáveis explicativas determinaram na expressão da variável MSC (variável dependente). O coeficiente de determinação obtido no presente estudo foi de $99,00 \%$, enquanto o efeito residual foi de $9,94 \%$. Os valores dos coeficientes de variação oscilaram de 7,10 - 21,20 $\%$, o que indica boa precisão experimental (Tabela 1).

A variável básica, MSC, foi correlacionada positivamente com os teores de $\mathrm{P}$ e $\mathrm{Zn}$ na parte aérea, SCC e NC. Não obstante, o CSSR e a MSSR correlacionaram-se negativamente com a MSC, indicando que o incremento do sistema radicular ocasionou redução na relação parte aérea-raiz. Esses resultados corroboraram aqueles apresentados nas superfícies de resposta, expostas nas Figuras 1 e 2. As maiores correlações positivas e diretas foram constatadas para os teores de $\mathrm{P}$ e $\mathrm{Zn}$ na parte aérea e para SCC. Esses resultados exprimiram que a aplicação de níveis adequados de $\mathrm{P}$ e $\mathrm{Zn}$ ao substrato possibilitou o aumento dos teores de ambos os elementos no tecido vegetal, incrementando a parte aérea, nesse caso, representada pelas seguintes variáveis: MSC (variável básica) e SCC (variável explicativa) (Tabela 1$)$.

Observou-se que o teor de P na parte aérea foi correlacionado em $98,70 \%$ com a MSC, dos quais, $19,50 \%$ foram efeitos indiretos via teor de $\mathrm{Zn}$ na parte aérea. $\mathrm{O}$ teor de $\mathrm{Zn}$ na parte aérea foi correlacionado em $97,80 \%$ com a MSC, dos quais $20,90 \%$ foram resultantes de efeito direto dessa variável explicativa sobre a MSC. O SCC correlacionou-se em 98,30\% com a MSC, em que apenas $18,90 \%$ da correlação ocorreu devido a efeitos diretos. O NC correlacionouse em $95,00 \%$ com a MSC, sendo que a maior parte dos efeitos de associação $(56,00 \%)$ foram efeitos indiretos via teor de $\mathrm{Pe} \mathrm{Zn}$ na parte aérea, e via SCC (Tabela 1).

Tanto o CSSR quanto a MSSR correlacionaram-se negativamente com a MSC. O CSSR correlacionou-se em $-59,20 \%$, com a MSC, dos quais $-15,60 \%$ foram devido a efeitos diretos. Já para a MSSR, a correlação negativa foi, ainda, mais expressiva, resultando em $94,70 \%$, dos quais $37,00 \%$ ocorreram devido a efeitos indiretos via teor de $\mathrm{P} \mathrm{e}$ $\mathrm{Zn}$ na parte aérea. Possivelmente, teores acentuados de $\mathrm{P}$ e Zn na parte aérea ocasionaram aumento da relação parte aérea-raiz, sendo que a situação recíproca resultou em efeitos contrários. Segundo Kurek et al. (2001), uma correlação negativa (caracteres em sentido contrário) indica que o aumento de uma variável corresponde à diminuição da outra. Esses autores acrescentam, ainda, que este fato pode ser atribuído à competição intrínseca da planta por fotoassimilados e aos fatores ambientais (Tabela 1). 

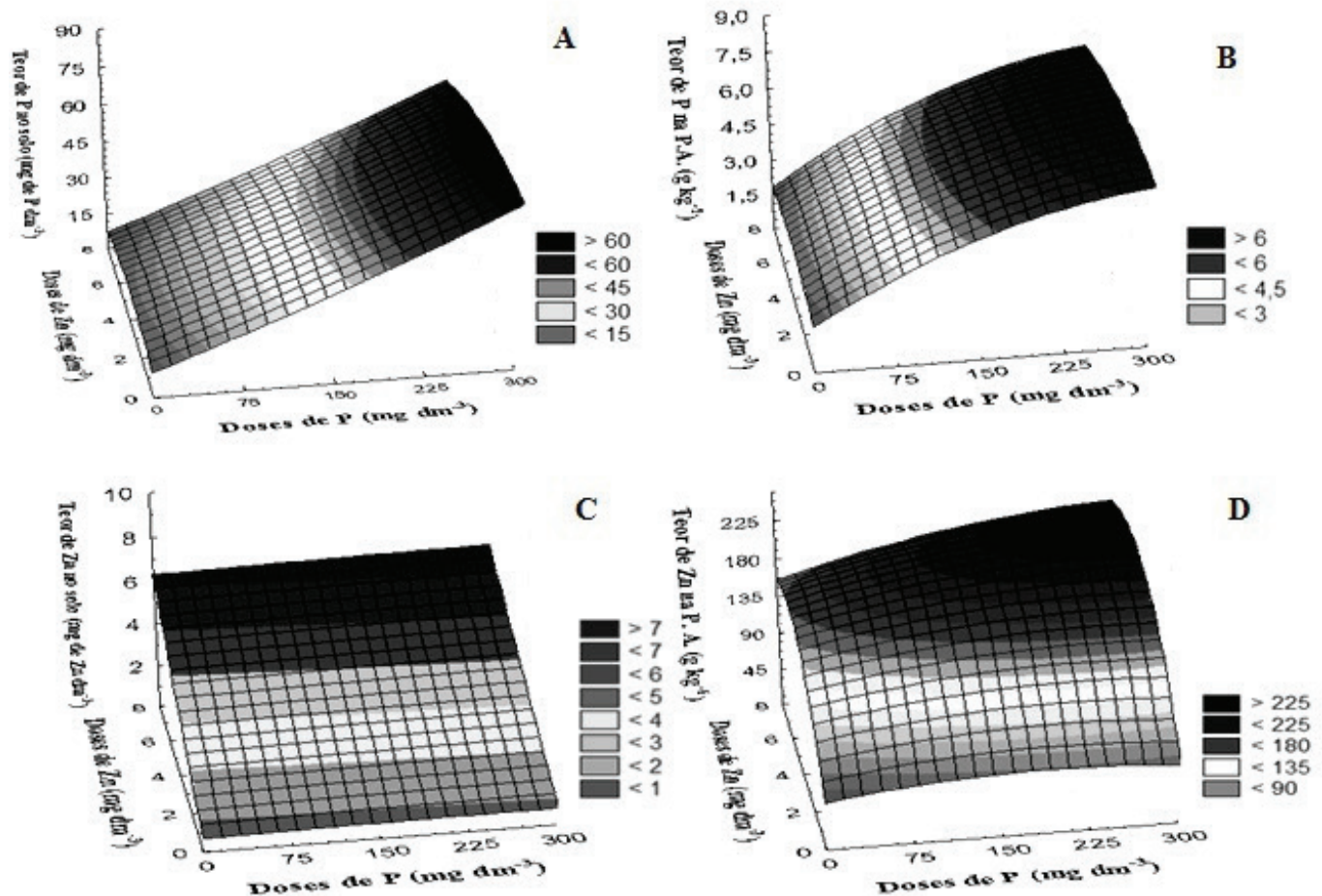

FIGURA 1 - Influência de doses de P e Zn sobre o teor de ambos os nutrientes em plantas de pitaia com 180 dias de idade. 1A: teor de $\mathrm{P}$ no substrato $\left[\hat{\mathrm{Y}}=10,1475+0,1667(\mathrm{P})+0,000064406(\mathrm{P})^{2}\right.$ $\left.+2,1916(\mathrm{Zn})-0,3188(\mathrm{Zn})^{2}-0,0008(\mathrm{P})(\mathrm{Zn}) ; \mathrm{R}^{2}=0,9199 *\right] ; 1 \mathrm{~B}$ : teor de $\mathrm{P}$ na parte aérea $\left[\hat{\mathrm{Y}}=1,9173+0,0267(\mathrm{P})-0,000037689(\mathrm{P})^{2}+0,2273(\mathrm{Zn})-0,0300(\mathrm{Zn})^{2}+0,0002(\mathrm{P})(\mathrm{Zn})\right.$ $\left.\mathrm{R}^{2}=0,8926^{*}\right]$; 1C: teor de $\mathrm{Zn}$ no substrato $\left[\hat{\mathrm{Y}}=0,6310+0,0008(\mathrm{P})-0,0000008127(\mathrm{P})^{2}+\right.$ $\left.0,6694(\mathrm{Zn})+0,0048(\mathrm{Zn})^{2}+0,000094(\mathrm{P})(\mathrm{Zn}) ; \mathrm{R}^{2}=0,8530 *\right] ; 1 \mathrm{D}$ teor de $\mathrm{Zn}$ na parte aérea $\left[\hat{\mathrm{Y}}=57,4373+0,2032(\mathrm{P})-0,0005(\mathrm{P})^{2}+38,2526(\mathrm{Zn})-3,2622(\mathrm{Zn})^{2}-0,0237(\mathrm{P})(\mathrm{Zn}) ; \mathrm{R}^{2}=\right.$ 0,6919*]. Universidade Federal do Ceará. Fortaleza-CE, 2013. 

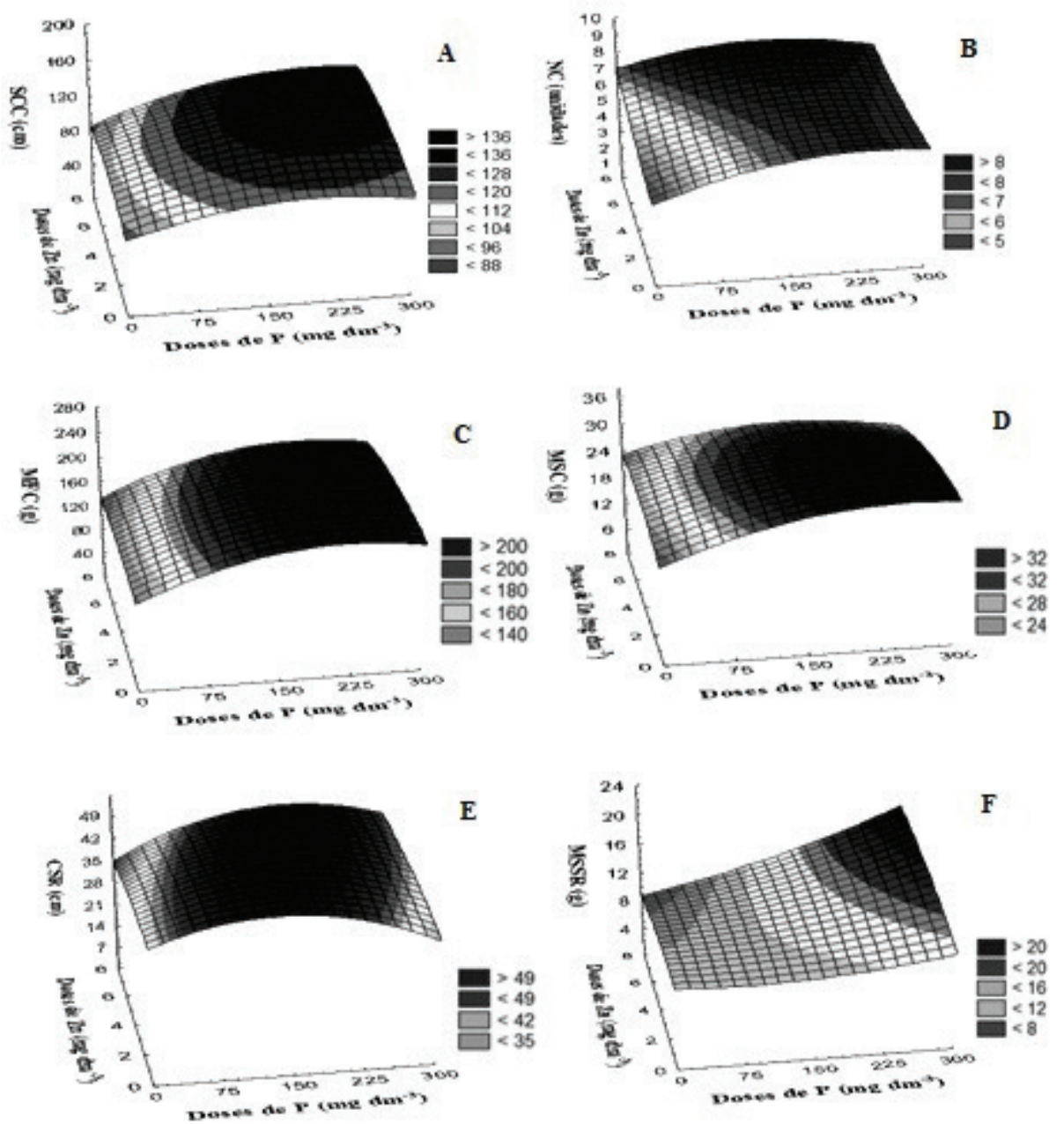

FIGURA 2 - Influência de doses de P e Zn sobre as características fenológicas de plantas de pitaia com 180 dias de idade. 2A: somatório do comprimento dos cladódios $[\hat{\mathrm{Y}}=85,1393+0,3193(\mathrm{P})$ - 0,0008(P) $\left.)^{2}+5,7884(\mathrm{Zn})-0,7230(\mathrm{Zn})^{2}+0,0107(\mathrm{P})(\mathrm{Zn}) ; \mathrm{R}^{2}=0,3515^{*}\right]$ 2B: número de cladódios $\left[\hat{\mathrm{Y}}=4,9471+0,0189(\mathrm{P})-0,00004(\mathrm{P})^{2}+0,2254(\mathrm{Zn})+0,0009(\mathrm{Zn})^{2}-0,0006(\mathrm{P})(\mathrm{Zn})\right.$; $\left.\mathrm{R}^{2}=0,3367^{*}\right]$; 2C: massa fresca dos cladódios $\left[\hat{\mathrm{Y}}=140,5957+0,6446(\mathrm{P})-0,0015(\mathrm{P})^{2}+\right.$ 3,9854(Zn) $\left.-0,6304(\mathrm{Zn})^{2}+0,0017(\mathrm{P})(\mathrm{Zn}) ; \mathrm{R}^{2}=0,4622 *\right]$; 2D: massa seca dos cladódios $[\hat{\mathrm{Y}}=$ $\left.22,0657+0,0848(\mathrm{P})-0,0002(\mathrm{P})^{2}+1,0129(\mathrm{Zn})-0,1116(\mathrm{Zn})^{2}-0,0029(\mathrm{P})(\mathrm{Zn}) ; \mathrm{R}^{2}=0,3702 *\right]$; 2E: comprimento do sistema radicular $\left[\hat{\mathrm{Y}}=41,9214+0,1026(\mathrm{P})-0,0004(\mathrm{P})^{2}-0,0682(\mathrm{Zn})\right.$ - 0,1018 $\left.(\mathrm{Zn})^{2}+0,0056(\mathrm{P})(\mathrm{Zn}) ; \mathrm{R}^{2}=0,4860^{*}\right] ; \mathbf{2 F}$ : massa seca do sistema radicular $[\hat{\mathrm{Y}}=$ $11,2671-0,0069(\mathrm{P})+0,000044441(\mathrm{P})^{2}+0,0554(\mathrm{Zn})-0,0491(\mathrm{Zn})^{2}+0,0035(\mathrm{P})(\mathrm{Zn}) ; \mathrm{R}^{2}=$ 0,4029*]. Universidade Federal do Ceará. Fortaleza-CE, 2013. 
TABELA 1- Estimativas dos efeitos diretos e indiretos que envolvem a variável principal massa seca de cladódios (MSC) e as variáveis independentes explicativas teor de fósforo (PPA) e zinco na parte aérea (ZnPA), somatório do comprimento ( $\mathrm{SCC}$ ) e número de cladódios $(\mathrm{NC})$, comprimento (CSSR) e massa seca do sistema radicular (MSSR) mensurado num acesso de pitaia (Hylocereus undatus) avaliado quanto a doses crescentes de fósforo e zinco. Fortaleza-CE, Brasil, 2013.

Caractere

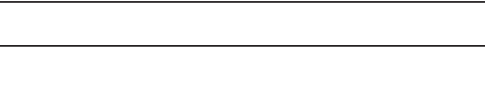

Efeitos de Associação $\quad$ Estimativa

Direto sobre MSC 0,193

Fósforo na parte aérea (PPA) Indireto via $\mathrm{ZnPA}$ 0,195 Indireto via SCC $\quad 0,189$ Indireto via NC $\quad 0,160$ Indireto via CSSR $\quad 0,071$ Indireto via MSR $\quad 0,170$

\begin{tabular}{lll} 
& Total & 0,987 \\
\hline \multirow{2}{*}{ Zinco na parte aérea (ZnPA) } & Direto sobre MSC & 0,209 \\
& Indireto via PPA & 0,180 \\
& Indireto via SCC & 0,174 \\
& Indireto via NC & 0,140 \\
& Indireto via CSSR & 0,116 \\
& Indireto via MSSR & 0,148
\end{tabular}

\begin{tabular}{|c|c|c|}
\hline \multirow{7}{*}{ Comprimento de cladódios (SCC) } & Direto sobre MSC & 0,189 \\
\hline & Indireto via PPA & 0,193 \\
\hline & Indireto via $\mathrm{ZnPA}$ & 0,193 \\
\hline & Indireto via NC & 0,160 \\
\hline & Indireto via CSSR & 0,067 \\
\hline & Indireto via MSR & 0,171 \\
\hline & Total & 0,983 \\
\hline \multirow{7}{*}{ Número de cladódios (NC) } & Direto sobre MSC & 0,162 \\
\hline & Indireto via PPA & 0,191 \\
\hline & Indireto via $\mathrm{ZnPA}$ & 0,181 \\
\hline & Indireto via SCC & 0,188 \\
\hline & Indireto via CSSR & 0,049 \\
\hline & Indireto via MSSR & 0,172 \\
\hline & Total & 0,950 \\
\hline \multirow{7}{*}{ Comprimento de raiz (CSSR) } & Direto sobre MSC & $-0,156$ \\
\hline & Indireto via PPA & $-0,088$ \\
\hline & Indireto via $\mathrm{ZnPA}$ & $-0,156$ \\
\hline & Indireto via SCC & $-0,082$ \\
\hline & Indireto via $\mathrm{NC}$ & $-0,050$ \\
\hline & Indireto via MSSR & $-0,052$ \\
\hline & Total & $-0,592$ \\
\hline \multirow{7}{*}{ Massa seca de raiz (MSSR) } & Direto sobre MSC & $-0,172$ \\
\hline & Indireto via PPA & $-0,190$ \\
\hline & Indireto via $\mathrm{ZnPA}$ & $-0,180$ \\
\hline & Indireto via SCC & $-0,187$ \\
\hline & Indireto via NC & $-0,162$ \\
\hline & Indireto via CSSR & $-0,047$ \\
\hline & Total & $-0,947$ \\
\hline Coeficiente de determinação & & 0,990 \\
\hline Valor de $k$ usado na análise & & 0,0488 \\
\hline Efeito da variável residual & & 0,0994 \\
\hline
\end{tabular}




\section{CONCLUSÕES}

A aplicação de $\mathrm{P}$ e Zn, e a interação fósforozinco afetam a disponibilidade de ambos os nutrientes no substrato, no sistema radicular e na parte aérea, influenciando, assim, o crescimento inicial de plantas de pitaia. Os rendimentos mais satisfatórios ocorrem quando há $60-75 \mathrm{mg}$ de $\mathrm{P} \mathrm{dm}^{-3}$ e $3,0-4,0 \mathrm{mg}$ de $\mathrm{Zn} \mathrm{dm}{ }^{-3}$ no substrato e $4,5-6,0 \mathrm{~g} \mathrm{~kg}^{-1}$ de P e $150 \mathrm{mg}$ de $\mathrm{Zn} \mathrm{kg}^{-1}$ na parte aérea.

\section{AGRADECIMENTOS}

Agradecemos à CAPES, pelo auxílio financeiro (Projeto 23038.006862/2010-02 - Edital 029/2010).

\section{REFERÊNCIAS}

ALMEIDA JÚNIOR, A. B.; OLIVEIRA, F. A.; MEDEIROS, J. F.; OLIVEIRA, M. K. T. Efeitos de doses de fósforo no desenvolvimento inicial da mamoneira. Revista Caatinga, Mossoró, v. 22, p. 217-221, 2009.

BATISTA, M . A. V.; PRADO, R. M.; LEITE, G. A. Resposta de mudas de goiabeira a aplicação de fósforo. Bioscience Journal, Uberlândia, v. 27, p, $635-641,2011$.

CESSA, R. M. A.; MOTA, J. H.; MELO, E. P. Produção de capuchinha cultivada em vaso com diferentes doses de fósforo e potássio em casa de vegetação. Global Science and Technology, Rio Verde, v. 2, p. 1-7, 2009.

COIMBRA, J. L. M.; BENIN, G.; VIEIRA, E. A.; OLIVEIRA, A. C.; CARVALHO, F. I. F.; GUIDOLIN, A. F.; SOARES, A. P. Consequências da multicolinearidade sobre a análise de trilha em canola. Ciência Rural, Santa Maria, v. 35, p. $347-$ $352,2005$.

CORRÊA, J. L.; MAUAD, M.; ROSELEM, C. A. Fósforo no solo e desenvolvimento de soja, influenciados pela adubação fosfatada e cobertura vegetal. Pesquisa Agropecuária Brasileira, Brasília, v. 39, p. 1.231-1.237, 2004.

CRUSCIOL, C. A. C.; MAUAD, M.; ALVAREZ, R. C. F.; LIMA, E. V.; TIRITAN, C. S. Doses de fósforo e crescimento radicular de cultivares de arroz de terras altas. Bragantia,Campinas, v. 64, p. 643-649, 2005.
DAVID, M. A.; MENDONÇA, V.; REIS, L. L. dos; SILVA, E. A. da; TOSTA, M. da S.; FREIRE, P. de A. Efeito de doses de superfosfato simples e de matéria orgânica sobre o crescimento de mudas de maracujazeiro-amarelo. Pesquisa Agropecuária Tropical, Goiânia, v. 38, p. 147-152, 2008.

DONADIO, L. C. Pitaya. Revista Brasileira de Fruticultura, Jaboticabal, v. 31, n. 3, p. 637-929, 2009.

FERNANDES, A. R.; PAIVA, H. N.; CARVALHO, J. G.; MIRANDA, J. R. P. Crescimento e absorção de nutrientes por mudas de freijó (Cordia goeldiana Huber) em função de doses de fósforo e de zinco. Revista Árvore, Viçosa, MG, v. 31, p.599-608, 2007.

GOLDSTEIN, G.; ORTEGA, J. K. E.; NERD, A.; NOBEL, P. S. Patterns of water potential components for the Crassulacean acid metabolism plant Opuntia ficus-indica when well-watered or droughted. Plant Physiology, New York, v. 95, p. 274-280, 1991.

KUREK, A. J.; CARVALHO, F. I. F.; ASSMANN, I. C.; MARCHIORO, V. S.; CRUZ, P. J. Análise de trilha como critério de seleção indireta para rendimento de grãos em feijão. Revista Brasileira de Agrociência, Pelotas, v. 7, p. 29-32, 2001.

LIMA, R. A. F.; MENDONÇA, V.; TOSTA, M. S.; REIS, L. L.; BISCARO, G. A.; CHAGAS, E. A. Fósforo e zinco no crescimento de mudas de maracujazeiro-amarelo. Pesquisa Agropecuária Tropical, Goiânia, v. 37, p. 251-256, 2007.

MARTINEZ, H. E. P.; ZABINI, A. V.; FRANCO, I. A. L.; NOVAIS, R. F. Translocação e compartimentalização de $\mathrm{Zn}$ em função de doses aplicadas em feijoeiro e cafeeiro via radicular. Ciência Rural, Santa Maria, v.35, p. 491-497, 2005.

MOREIRA, R. A; RAMOS, J. D.; MARQUES, V. B.; ARAÚJO, N. A.; MELO, P. C. Crescimento de pitaia- vermelha com adubação orgânica e granulado bioclástico. Ciência Rural, Santa Maria, v. 41, p. 785-788, 2011.

MUNER, L. H.; RUIZ, H. A.; VENEGAS, V. H. A.; NEVES, J. C. L.; FREIRE, F. J.; FREIRE, M. B. G. dos S. Disponibilidade de zinco para milho em resposta à localização de fósforo no solo. Revista Brasileira de Engenharia Agrícola e Ambiental, Campina Grande, v. 15, p. 29-38, 2011. 
ORTIZ-HERNÁNDEZ, Y. D.; CARRILLOSALAZAR, J.A. Pitahaya (Hylocereus spp.): a short review. Comunicata Scientiae, Bom Jesus, v. 3, p. $220-237.2012$.

RAMOS, J. P. ; LEITE, M. L. M. V.; OLIVEIRA JÚNIOR, S.; NASCIMENTO, J. P.; SANTOS, E. M. Crescimento vegetativo de Opuntia ficus-indica em diferentes espaçamentos de plantio. Revista Caatinga, Mossoró, v. 24, p. 41 - 48, 2011.

ROSOLEM, C. A.; WITACKER, J. P. T.; VANZOLINI, S.; RAMOS, V. J. Significance of root growth on cotton nutrition in an acidic low-P soil. Plant and Soil, Dordrecht, v. 212, p.185-190, 1999.

SARTORI, R. H.; BOARETTO, A. E.; VILLANUEVA, F. C. A.; FERNADES, H. M. G. Absorção radicular e foliar de $\mathrm{Zn}$ e sua redistribuição em laranjeiras. Revista Brasileira de Fruticultura, Jaboticabal, v. 30, p. 523-528, 2008.
SILVA, S. A.; CARVALHO, F. I. F.; NEDEL, J. L.; CRUZ, P. J.; SILVA, J. A. G.; CAETANO, V. R.; HARTWIG, I.; SOUSA, C. S. Análise de trilha para os componentes de rendimento de grãos em trigo. Bragantia, Campinas, v. 64, p. 191-196, 2005.

TELES, M. M.; SANTOS, M. V. F.; JÚNIOR, J. C. B. D.; NETO, E. B.; FERREIRA, R. L. C.; LUCENA, J. E. C.; LIRA, M. A. Efeitos da adubação e de nematicida no crescimento e na produção da palma forrageira (Opuntia ficus indica Mill) cv. Gigante. Revista Brasileira de Zootecnia, Viçosa, MG, v. 31, p. 52-60. 2002.

WRIGHT, S. Correlation and causation. Journal of Agricultural Research, Lahore, v. 20, p. 557-585, 1921. 